\title{
EMIGRACIÓN DE RECURSOS HUMANOS CALIFICADOS Y DESARROLLO EN AMÉRICA LATINA Y EL CARIBE: UNA MIRADA DESDE LA GEOGRAFÍA
}

\author{
MIGRATION OF SKILLED HUMAN RESOURCES AND DEVELOPMENT IN LATIN \\ AMERICA AND THE CARIBBEAN: A VIEW THROUGH THE GEOGRAPHY
}

\author{
Mg. Lcdo. Eloy Montes Galbán \\ Departamento de Geografía/ \\ Centro de Estudios Geográficos \\ Universidad del Zulia \\ Doctorando en Geografía - UNNE \\ E-mail: eloyead@yahoo.com
}

La presente contribución fue realizada en el marco del curso de posgrado "El análisis demográfico como herramienta para el estudio de la población y el desarrollo" perteneciente al Doctorado en Geografía de la Universidad Nacional del Nordeste, Agosto de 2014.

\section{Resumen}

El presente estudio tiene por objetivo determinar las unidades espaciales (países) que cumplen con cada una de las relaciones establecidas para el nivel bivariado entre la emigración de recursos humanos calificados y desarrollo en América Latina y el Caribe (ALC). La metodología implementada constó de dos fases, en la primera se creó una base de datos geográfica digital, seleccionando y ordenando los indicadores a ser correlacionados como variables independientes (Esperanza de vida al nacer; Ayuda Oficial al Desarrollo neta recibida y el Índice de Globalización) y dependiente (Emigración de recursos humanos calificados), para esto se contó con estadísticas del año 2008; en la segunda fase se aplicó la técnica de Análisis Exploratorio de Datos Espaciales(ESDA, Exploratory Spatial Data Analysis)específicamente procedimientos de análisis bivariado (2D) con el apoyo del software GeoDa. De los resultados del ESDA se deriva: en cuanto a la relación entre el índice de esperanza de vida al nacer y la tasa de emigración calificada en ALC, la tendencia es que a menor índice de esperanza de vida al nacer mayor tasa de emigración calificada (TEC); en cuanto a la relación entre la Ayuda Oficial al Desarrollo con la TEC la correlación es positiva; del resultado de la tercera y última correlación se concluye que a menores niveles en los índices de globalización mayores TEC.Los países con una situación más favorable son: Argentina, Chile, Colombia, Ecuador, Perú, Uruguay, Costa Rica, México y Panamá; asimismo, los que presentan una situación menos favorable son: Surinam, Dominica, Haití, San Cristóbal y Nieves, San Vicente y Granadinas.

Palabras Clave

Emigración calificada, Desarrollo, América Latina y el Caribe, Análisis Exploratorio de Datos Espaciales.

\begin{abstract}
This studyaims to determine the spatial units(countries) which observeeachof the relations forbivariate level between the emigration of skilled human resources and developmentin Latin America and the Caribbean (LAC). The implemented methodology consisted of two phases, at first, a base of digital geographic data was created by selecting and ordering the indicators to be correlated such as independent variables (life expectancy at birth, net Official Development Assistance received and the Globalization Index) dependent variable(Emigration of skilled humanresources), to achieve this goal, 2008 statistics were applied; in the second phase the Exploratory Spatial Data Analysis technique(ESDA, Exploratory Analysis Spatial Data) was applied specificallybivariate analysis
\end{abstract}

Publicado en formato digital: Mg. Lcdo. Eloy Montes Galbán. EMIGRACIÓN DE RECURSOS HUMANOS CALIFICADOS Y DESARROLLO EN AMÉRICA LATINA Y EL CARIBE: UNA MIRADA DESDE LA GEOGRAFÍA. Revista Geográfica Digital. IGUNNE. Facultad de Humanidades. UNNE. Año 11. No 22. Julio Diciembre. 2014. ISSN 1668-5180 Resistencia, Chaco. 
procedures (2D) with the support from the GeoDa software. From the results of ESDA is derived: as regards to the relationship between the rate of life expectancy at birth and the rate of skilled emigration in LAC, the trend shows that the lower rate of life expectancy at birth increased rate of skilled emigration (TEC); regarding the relationship between Official Development Assistance with ECT the correlation is positive; through the result of the third and the last correlation it is concluded that at lower globalization levels higher TEC rates. Countries with a more favorable situation are: Argentina, Chile, Colombia, Ecuador, Peru, Uruguay, Costa Rica, Mexico and Panama; also, those with a less favorable situation are: Suriname, Dominica, Haiti, Federation of Saint Christopher and Nevis, St. Vincent and Grenadines.

\section{Keywords}

Skilled migration, Development, Latin America and Caribbean, Exploratory Spatial Data Analysis.

Publicado en formato digital: Mg. Lcdo. Eloy Montes Galbán. EMIGRACIÓN DE RECURSOS HUMANOS CALIFICADOS Y DESARROLLO EN AMÉRICA LATINA Y EL CARIBE: UNA MIRADA DESDE LA GEOGRAFÍA. Revista Geográfica Digital. IGUNNE. Facultad de Humanidades. UNNE. Año 11. No 22. Julio Diciembre. 2014. ISSN 1668-5180 Resistencia, Chaco.

En: http://hum.unne.edu.ar/revistas/geoweb/default.htm 


\section{Introducción}

La Organización Internacional para las Migraciones define este proceso como "el movimiento de una persona o grupo de personas de una unidad geográfica hacia otra a través de una frontera administrativa o política con la intención de establecerse de manera indefinida o temporal en un lugar distinto a su lugar de origen" sin embargo en el marco de las nuevas características que presenta la migración internacional (1)hoy día se entiende que, el tipo de población que migra es heterogénea, con diversas características culturales, educativas y económicas. La mayoría de los estudios concebían a los migrantes como un todo homogéneo, que los suponía hombres en su mayoría, trabajadores -no calificados-, impulsados por motivos económicos, cuyos movimientos se asumían como permanentes o definitivos, no dando espacio a las migraciones temporales, circulares o de retorno.

Una de las tendencias que caracterizan el escenario contemporáneo de los movimientos internacionales de población, es la migración de recursos humanos con alta calificación, entendiendo por alta calificación aquellos individuos migrantes dotados de cierta preparación, ya sea por el nivel educativo logrado, por el tipo de ocupación que posee o en referencia a ambos (SELA, 2009). En el presente estudio se define a los migrantes calificados como aquellas personas que han logrado una educación correspondiente a estudios de tercer nivel y que nacieron en otro país distinto al de residencia.

La migración en general, y la migración calificada en particular, han sido estudiadas en la mayoría de los casos como una categoría homogénea, cuando en realidad presentan ciertas particularidades. Estas diferencias en la migración internacional contemporánea con diversidad de factores incidiendo en su comportamiento y con un origen multicausal, requieren no sólo de investigaciones y estudios específicos sino también de un marco teórico amplio que posibilite las explicaciones.

Según el Banco Mundial (2009) esta tendencia creciente de la migración calificada internacional tiene al menos dos factores explicativos: por un lado, el proceso globalizador, por el otro, las políticas de inmigración. Asimismo el:

"aumento generalizado de personas con mayor calificación, una mayor demanda laboral de ciertas profesiones y de sectores específicos de la economía aunado apolíticas favorecedoras para el traslado de personas con altos niveles formativos, explican la tendencia creciente a emigrar de esta mano de obra" (SELA, 2010, p. 8)

Entre las consecuencias que se asocian con la emigración de recursos humanos calificados, está el debilitamiento de la población con alta formación, que también deriva en la pérdida de recursos invertidos en educación, circunstancias que repercuten sobre el crecimiento económico de los países emisores (Martínez Pizarro, 2006). Por su naturaleza selectiva, el impacto negativo de la emigración de recuso humano calificado tiene su principal efecto en el país de origen, contribuyendo en algunos casos a la profundización del subdesarrollo y al incremento de las desigualdades.

Una de las regiones que presenta mayor dinamismo con respecto a la migración calificada en las últimas décadas es América Latina y el Caribe (ALC), entre 1990 y 2008 (figura 1), la población con origen en ALC y residente en algún país de la Organización para la Cooperación y el Desarrollo Económico(OCDE) se incrementó en un 164 \% (SELA, 2010).

Publicado en formato digital: Mg. Lcdo. Eloy Montes Galbán. EMIGRACIÓN DE RECURSOS HUMANOS CALIFICADOS Y DESARROLLO EN AMÉRICA LATINA Y EL CARIBE: UNA MIRADA DESDE LA GEOGRAFÍA. Revista Geográfica Digital. IGUNNE. Facultad de Humanidades. UNNE. Año 11. No 22. Julio Diciembre. 2014. ISSN 1668-5180 Resistencia, Chaco. 
1990

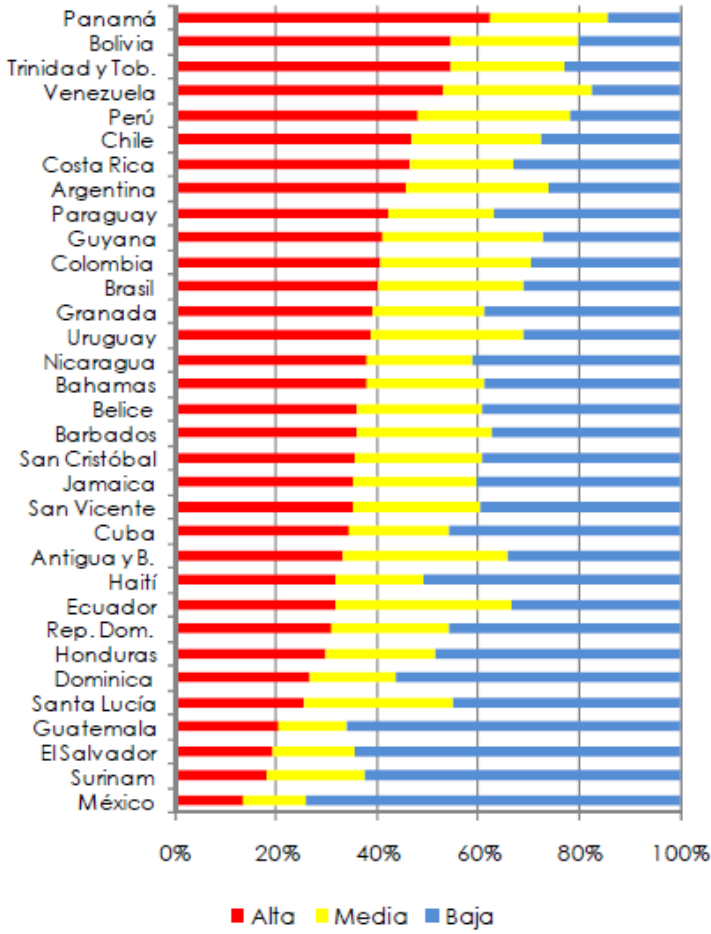

2008

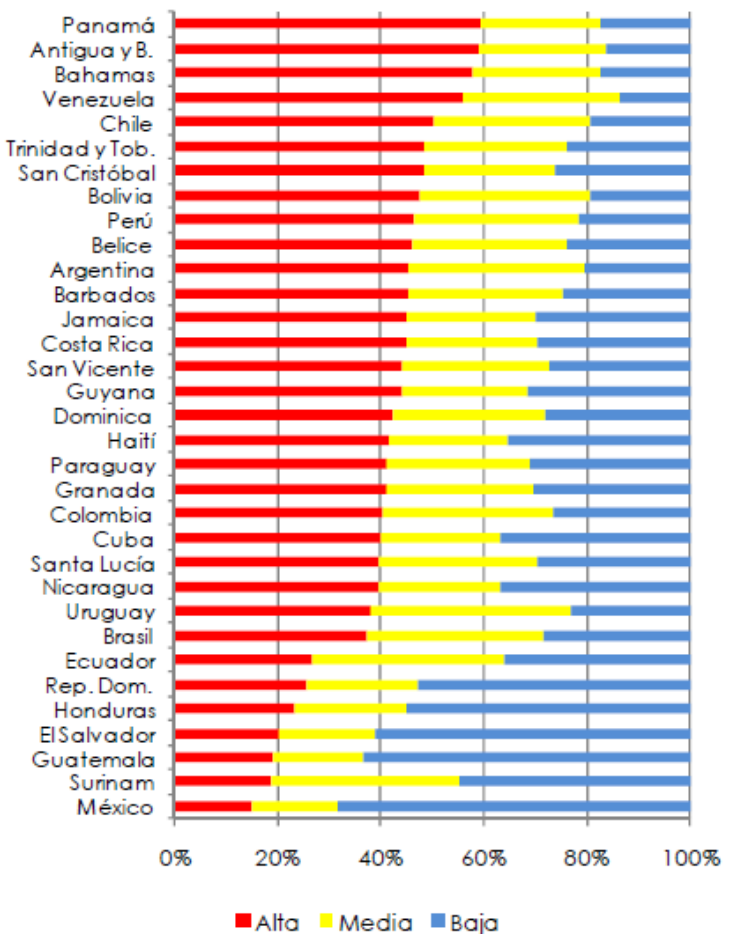

Figura 1. Población de migrantes de 25 años y más, residentes en los países de la OCDE por nivel de calificación según país de origen de ALC, 1990 y 2008.

Fuente: SELA (2010) con base en Docquier, Lowell y Marfouk (2009)

La cifra de 2008 es una estimación con base a las tasas de crecimiento observadas entre 1990 y 2000.

Si la migración no puede concebirse como un fenómeno que solo se origina por factores económicos como: pobreza, insuficiencia o desigualdad de ingresos y bajo nivel de desarrollo económico; entonces es mucho más adecuado entenderla como un fenómeno multicausal, esto quiere decir que al hablar de desarrollo no se puede asumir la postura tradicional que solo lo relaciona con variables e indicadores económicos. En este caso se debe entender el desarrollo como un fenómeno que involucra la dinámica de dimensiones: económica, social, cultural, idiosincrática, política entre otras.

En consecuencia se puede afirmar que la relación entre la migración internacional en general y específicamente la migración de personas con altos niveles de escolaridad y el desarrollo humano es heterogénea y compleja. De alguna manera lo que se busca con el presente estudios es arrojar luces sobre esta realidad tan compleja, a través de la observación de patrones de asociación entre las variables de emigración calificada el desarrollo y sus manifestaciones espaciales.

Para la delimitación espacio-temporal del presente trabajo se partió de la fuente de información de la variable dependiente (emigración de recursos humanos calificado) con la que se contaba, la base de datos elaborada por Docquier,Lowell y Marfouk (2009) sobre la población de migrantes internacionales de 25 años y más, residentes en los países de la Organización para la Cooperación y el Desarrollo Económico (OCDE)(2)según nivel de escolaridad, para los años 1990 y 2000, y a partir

Publicado en formato digital: Mg. Lcdo. Eloy Montes Galbán. EMIGRACIÓN DE RECURSOS HUMANOS CALIFICADOS Y DESARROLLO EN AMÉRICA LATINA Y EL CARIBE: UNA MIRADA DESDE LA GEOGRAFÍA. Revista Geográfica Digital. IGUNNE. Facultad de Humanidades. UNNE. Año 11. No 22. Julio Diciembre. 2014. ISSN 1668-5180 Resistencia, Chaco.

En: http://hum.unne.edu.ar/revistas/geoweb/default.htm 
de allí las estimaciones realizadas para el año 2008 en un estudio del SELA (2010) que comprende 33 países de América Latina y el Caribe. (Figura 2)

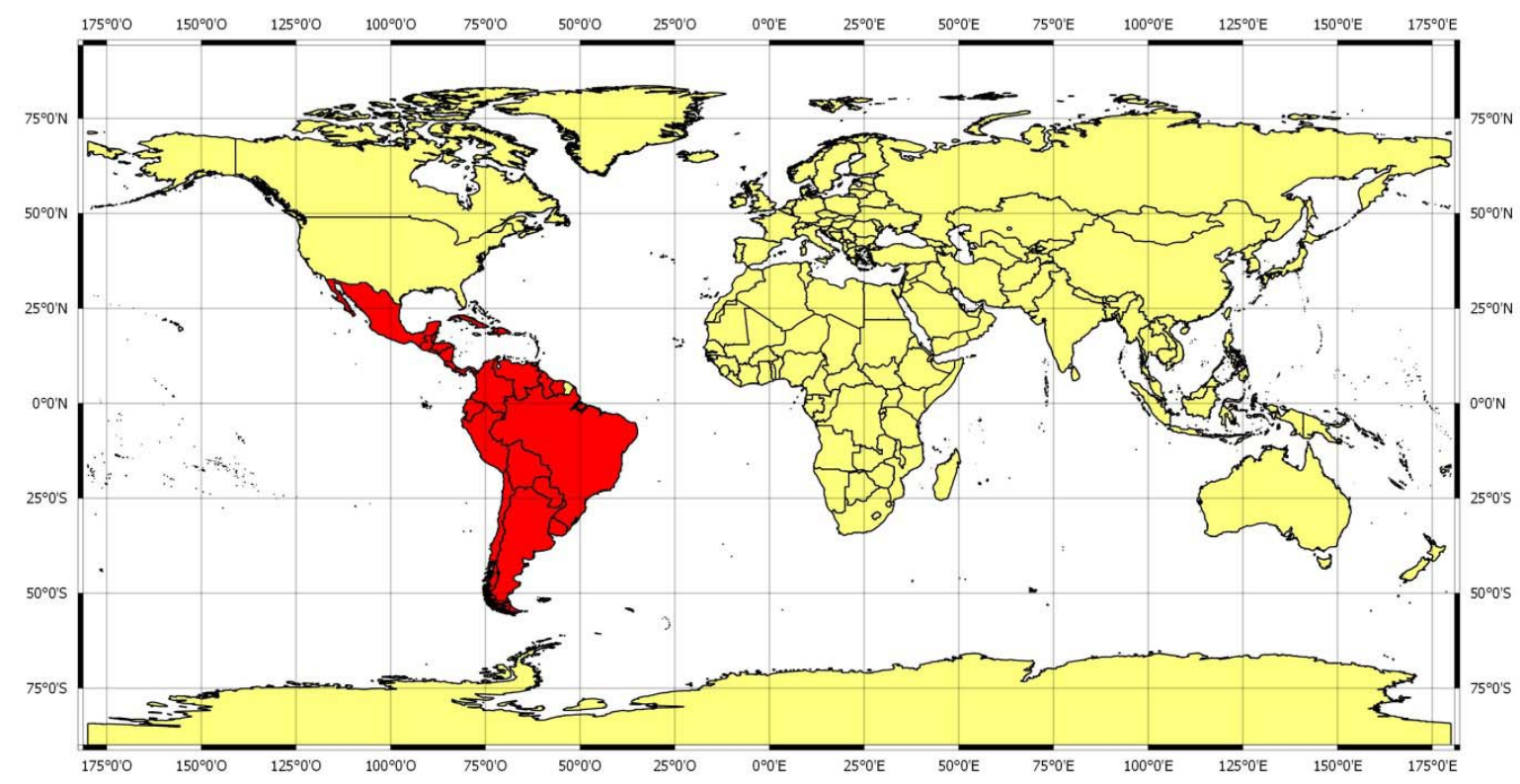

Figura 2. Situación relativa de los países de América Latina y el Caribe (ALC) considerados en el estudio.

Fuente: elaboración propia con el software QGIS 2.4. y en base a la cartográfica de Natural Earth versión 3.1.0.(2013).

El objetivo del presente trabajo es determinar las unidades espaciales (países) que cumplen con cada una de las relaciones establecidas para el nivel bivariado entre la emigración de recursos humanos calificados y desarrollo en América Latina y el Caribe.

\section{Metodología}

Se asumió una metodología orientada por un enfoque empirista-inductivo, que permite aproximar un diagnóstico de la situación actual en América Latina, para luego fundamentar futuras hipótesis de trabajo y propuestas conceptuales desde una perspectiva territorial. Específicamente en este caso se implementó la técnica del Análisis Exploratorio de Datos Espaciales (ESDA, ExploratorySpatial Data Analysis) que se encuentra enmarcada dentro del análisis exploratorio de datos (EDA, Exploratory Data Analysis), esta es una herramienta técnico-metodológica que permite realizar las primeras aproximaciones al estudio de la estructura de la información socio-espacial en un determinado territorio (Buzai y Baxendale, 2012), existen diversos antecedentes donde se evidencian las posibilidades técnicas actuales (Bosque y Moreno, 1994; Buzai y Baxendale, 2009).La aplicación del ESDA le permite al investigador descubrir errores en la codificación de los datos, determinar los casos anómalos, la posibilidad de comprobar supuestos (hipótesis) y aproximarse al comportamiento de cada variable (univariado) así como la relación que guarda con otras (bivariado o trivariado), esto último es el caso que nos ocupa en el presente estudio.

El diseño de la investigación consta de dos fases con sus respectivas actividades, la primera de ellas consiste en crear la base de datos geográfica digital, conformada por datos espaciales y atributos o temáticos. Para esto, previamente, se realizó una selección de cuatro dimensiones con sus respectivos indicadores para 33 países de América Latina y el Caribe, estos a su vez se dividieron en variables independientes y dependiente, entre las primeras se seleccionaron los

Publicado en formato digital: Mg. Lcdo. Eloy Montes Galbán. EMIGRACIÓN DE RECURSOS HUMANOS CALIFICADOS Y DESARROLLO EN AMÉRICA LATINA Y EL CARIBE: UNA MIRADA DESDE LA GEOGRAFÍA. Revista Geográfica Digital. IGUNNE. Facultad de Humanidades. UNNE. Año 11. № 22. Julio Diciembre. 2014. ISSN 1668-5180 Resistencia, Chaco.

En: http://hum.unne.edu.ar/revistas/geoweb/default.htm 
indicadores: Esperanza de vida al nacer; Ayuda Oficial al Desarrollo (ODA) neta recibiday el Índice de Globalización. En el caso de la variable dependiente está la emigración de recursos humanos calificados. Para todo esto se consultaron variadas fuentes que se describen en la tabla 1.

Tabla 1. Variables e indicadores correlacionados en el estudio, su descripción y fuente.

\begin{tabular}{|c|c|c|c|c|}
\hline $\begin{array}{c}\text { Tipo de } \\
\text { variable }\end{array}$ & Dimensión & Indicadores & Descripción & $\begin{array}{l}\text { Fuente de } \\
\text { los datos }\end{array}$ \\
\hline \multirow{3}{*}{$\begin{array}{l}\text { Variables } \\
\text { Independientes } \\
\text { (Beneficio) }\end{array}$} & $\begin{array}{l}\text { Desarrollo } \\
\text { humano }\end{array}$ & $\begin{array}{l}\text { Esperanza de } \\
\text { vida al nacer }\end{array}$ & $\begin{array}{l}\text { La esperanza de vida al nacer } \\
\text { indica la cantidad de años que } \\
\text { viviría un recién nacido si los } \\
\text { patrones de mortalidad vigentes } \\
\text { al momento de su nacimiento no } \\
\text { cambian a lo largo de la vida del } \\
\text { infante. }\end{array}$ & $\begin{array}{l}\text { Banco } \\
\text { Mundial } \\
(2008)\end{array}$ \\
\hline & $\begin{array}{l}\text { Económica } \\
\text { (flujos } \\
\text { financieros) }\end{array}$ & $\begin{array}{l}\text { Ayuda Oficial } \\
\text { al Desarrollo } \\
\text { (ODA) neta } \\
\text { recibida (\% del } \\
\text { INB) }\end{array}$ & $\begin{array}{l}\text { La asistencia oficial neta para el } \\
\text { desarrollo (AOD) comprende los } \\
\text { desembolsos de préstamos en } \\
\text { condiciones concesionarias } \\
\text { (netos de reembolsos del } \\
\text { principal) y las donaciones } \\
\text { otorgadas por organismos } \\
\text { oficiales de los miembros del } \\
\text { Comité de Asistencia para el } \\
\text { Desarrollo (CAD), por } \\
\text { instituciones multilaterales y por } \\
\text { países que no integran el CAD a } \\
\text { fin de promover el desarrollo y el } \\
\text { bienestar económico en los } \\
\text { países y territorios que figuran } \\
\text { en la lista del CAD de } \\
\text { destinatarios de AOD. Incluye } \\
\text { los préstamos que tienen un } \\
\text { elemento de donación de al } \\
\text { menos el } 25 \% \text { (calculado a una } \\
\text { tasa de descuento del 10\%). }\end{array}$ & $\begin{array}{l}\text { Banco } \\
\text { Mundial } \\
\text { (2008) }\end{array}$ \\
\hline & $\begin{array}{l}\text { Grado de } \\
\text { inserción de } \\
\text { los países } \\
\text { ALC con el } \\
\text { mundo global }\end{array}$ & $\begin{array}{l}\text { Índice de } \\
\text { Globalización }\end{array}$ & 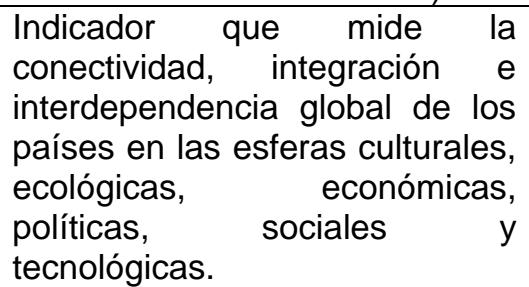 & $\begin{array}{l}\text { Instituto de } \\
\text { investigación } \\
\text { económica } \\
\text { Konjunkturfo } \\
\text { rschungsstel } \\
\text { le KOF } \\
(2011) \quad\end{array}$ \\
\hline $\begin{array}{l}\text { Variable } \\
\text { dependiente } \\
\text { (Costo) }\end{array}$ & $\begin{array}{l}\text { Socio } \\
\text { demográfica }\end{array}$ & $\begin{array}{l}\text { Emigración de } \\
\text { recursos } \\
\text { humanos } \\
\text { calificados }\end{array}$ & $\begin{array}{l}\text { Tasa de emigración de alta } \\
\text { calificación de } 25 \text { años y más, } \\
\text { con origen en ALC para el } 2008^{*} \\
\text { y residentes en los países de la } \\
\text { Organización para la } \\
\text { Cooperación y el Desarrollo } \\
\text { Económico (OCDE). }\end{array}$ & $\begin{array}{l}\text { SELA (2010) } \\
\text { con base en } \\
\text { Docquier, } \\
\text { Lowell y } \\
\text { Marfouk } \\
\text { (2009) }\end{array}$ \\
\hline
\end{tabular}

Fuente: elaboración propia.

*Las cifras son una estimación con base a las tasas de crecimiento observadas entre 1990 y 2000.

Publicado en formato digital: Mg. Lcdo. Eloy Montes Galbán. EMIGRACIÓN DE RECURSOS HUMANOS CALIFICADOS Y DESARROLLO EN AMÉRICA LATINA Y EL CARIBE: UNA MIRADA DESDE LA GEOGRAFÍA. Revista Geográfica Digital. IGUNNE. Facultad de Humanidades. UNNE. Año 11. № 22. Julio Diciembre. 2014. ISSN 1668-5180 Resistencia, Chaco.

En: http://hum.unne.edu.ar/revistas/geoweb/default.htm 
En una segunda fase del estudio, las Tecnologías de la Información Geográficas cumplen un papel fundamental como herramientas que han revolucionado los estudios espaciales del siglo XXI, ya no se puede concebir una disciplina en Geociencias que no aplique tan útiles técnicas (Cerezo y Col., 2013) dentro de esta gama de posibilidades se encuentran los software específicos como el GeoDa(3), con excelentes aptitudes para el desarrollo de cartografía temática y avances en el Análisis Exploratorio de Datos Espaciales (ESDA), posibilitando el análisis con el apoyo de gráficos interactivos (Buzai y col., 2013).

El análisis bivariado (2D) con el software GeoDa que nos ocupa en el presente trabajo se basa en procedimientos comparativos de variables, dicho procedimiento se apoya en el gráfico de dispersión con dos ejes de coordenadas, a partir del cual la nube de puntos formada por las mediciones individuales muestran una aproximación al tipo, tendencia e intensidad de la relación (Buzai y Baxendale, 2012).

El gráfico de dispersión 2D permite verificar las relaciones que se producen de forma lineal y con ello dos características esenciales: (a) el sentido de la relación, y (b) su intensidad. Aspectos que se hacen evidentes fácilmente a partir de la configuración gráfica. (Buzai y Baxendale, 2009).

Para la interpretación del gráfico (gráfico de dispersión en dos dimensiones)se deben tomar en cuenta los siguientes aspectos:

- Los resultados de cada variable quedan representados en un eje ortogonal $\left(90^{\circ}\right)$ y cada unidad espacial como un punto de localización $x-y$ a partir de sus valores de coordenadas en cada eje.

- Los datos de cada variable se transforman a puntajes estándar (Standardized Data) tomando cada uno de los ejes el sector central del gráfico apareciendo cuatro cuadrantes definidos a partir de las medias de ambas variables que toman valores cero (0).

- El cuadrante inferior izquierdo concentra unidades espaciales (en este caso países de ALC) con bajos valores en ambas variables, el cuadrante superior izquierdo con bajos valores en $x$ y altos en $y$, el cuadrante superior derecho con valores altos en ambas variables, $y$ el cuadrante inferior derecho con valores altos en $x$ y bajos en $y$ (figura 3 ).

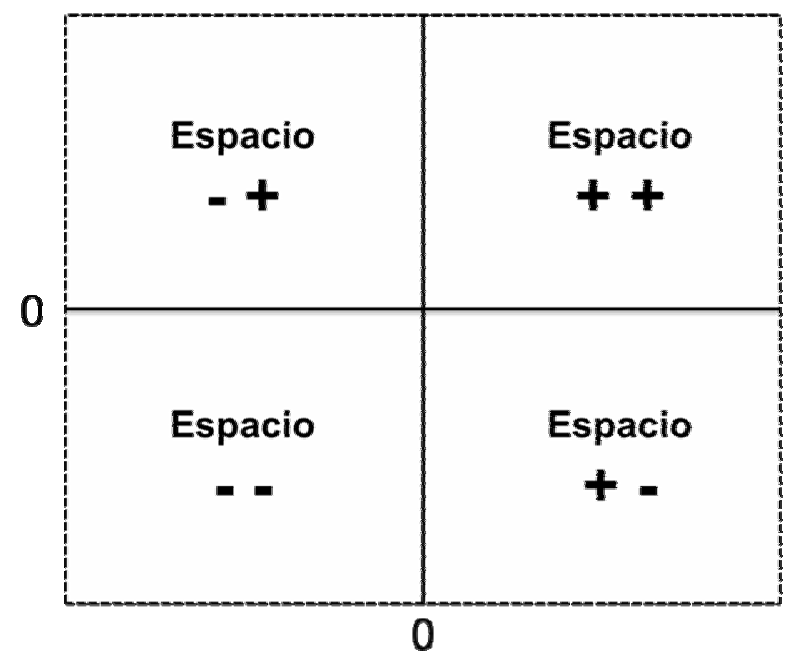

Figura 3. Espacio de relaciones bivariadas entre variables estandarizadas.

Fuente: elaboración propia en base a Buzai y Baxendale (2012) (Modificado)

Publicado en formato digital: Mg. Lcdo. Eloy Montes Galbán. EMIGRACIÓN DE RECURSOS HUMANOS CALIFICADOS Y DESARROLLO EN AMÉRICA LATINA Y EL CARIBE: UNA MIRADA DESDE LA GEOGRAFÍA. Revista Geográfica Digital. IGUNNE. Facultad de Humanidades. UNNE. Año 11. No 22. Julio Diciembre. 2014. ISSN 1668-5180 Resistencia, Chaco.

En: http://hum.unne.edu.ar/revistas/geoweb/default.htm 


\section{Resultados y discusión}

Los resultados se estructuraron en dos secciones, la primera consiste en una evaluación general que muestra el grado de asociación positiva o negativa entre las diversas variables de desarrollo y económicas con la tasa de emigración calificada; en la segunda sección se detalla aquellos países que se encuentran en situaciones más favorables y menos favorables en cuanto a las relaciones (correlación) estudiadas.

En la figura 4 se inicia (gráfico de la izquierda) con la relación entre la esperanza de vida al nacer (eje $x$ ) y la tasa de emigración calificada (eje $y$ ), claramente se observa como la nube de puntos formada tiene una tendencia descendente desde el espacio -+ al espacio +-, en el gráfico de dispersión se puede apreciar que el coeficiente de correlación para ambas variables es de $r=-0,475$. Este resultado indica que: (a) existe una relación entre las dos variables, (b) el sentido de la relación es inverso, es decir cuando aumentan /disminuyen los valores de una variable disminuyen /aumentan los valores de la otra, (c) el grado de la relación según Hernández et al. (2003) se considera como "correlación negativa media" ya que su valor está cercano al -0,50.

Si se considera que esta correlación es significativa, el resultado muestra que altas tasas de emigración calificada se asocian a índices más bajos de esperanza de vida en los países de ALC, según el informe del SELA (2010) hace mención al resultado contrario propuesto en el informe de Desarrollo Humano (2009), el cual indica que la migración en general puede llegar a impactar de manera positiva en la salud de la población de los países de origen.

El gráfico central de la figura 4, muestra la relación entre la Ayuda Oficial al Desarrollo (ODA) neta recibida (eje $x$ ) y la tasa de emigración calificada (eje $y$ ), claramente se observa como la nube de puntos formada tiene una tendencia ascendente desde el espacio -- al espacio ++, en el gráfico de dispersión se puede apreciar que el coeficiente de correlación para ambas variables es de $r=0,519$. Este resultado indica que: (a) existe una relación entre las dos variables, (b) el sentido de la relación es positiva, es decir cuando aumentan /disminuyen los valores de una variable aumentan /disminuyen los valores de la otra, (c) el grado de la relación según Hernández et al. (2003) se considera como "correlación positiva media" ya que su valor está cercano a 0,50.

La variable ODA presenta una correlación significativa con la tasa de emigración calificada, con signo positivo, al respecto los datos muestran que a mayor \% del INB de este flujo financiero se relaciona con tasas de emigración calificada también mayores. A pesar de la premisa asumida por muchos organismos internacionales que la asistencia para el desarrollo es considerada como una de las políticas más efectivas para reducir la migración internacional, se tienen evidencias empíricas que muestran que el crecimiento económico en muchos países no necesariamente es igual a menores tasas de emigración (Belloc, 2008; SELA, 2010); por el contrario, la migración se incrementa debido a que los individuos pueden afrontar los costos y riesgos de un desplazamiento internacional (SELA, 2010).

El gráfico de dispersión de la derecha (figura 4) muestra la relación entre el Índice de Globalización (KOF) (eje $x$ ) y la tasa de emigración calificada (eje $y$ ), se observa como la nube de puntos formada tiene una tendencia descendente desde el espacio -+ al espacio +-, en el gráfico se puede apreciar que el coeficiente de correlación para ambas variables es de $r=-0,565$. Este resultado indica que: (a) existe una relación entre las dos variables, (b) el sentido de la relación es inverso, es decir cuando aumentan /disminuyen los valores de una variable disminuyen /aumentan los valores de la otra, (c) el grado de la relación según Hernández et al. (2003) se considera como "correlación negativa media" por estar su valor cercano al -0,50.

Publicado en formato digital: Mg. Lcdo. Eloy Montes Galbán. EMIGRACIÓN DE RECURSOS HUMANOS CALIFICADOS Y DESARROLLO EN AMÉRICA LATINA Y EL CARIBE: UNA MIRADA DESDE LA GEOGRAFÍA. Revista Geográfica Digital. IGUNNE. Facultad de Humanidades. UNNE. Año 11. No 22. Julio Diciembre. 2014. ISSN 1668-5180 Resistencia, Chaco. 
Esta tercera y última variable, es un indicador que mide tres dimensiones de la globalización: económica, social y política, los resultados de la correlación entre el índice de globalización y la tasa de emigración calificada, indican que a menor nivel del índice de globalización mayores tasas de emigración calificada. Existen evidencias de estudios realizados en países asiáticos que han demostrado que al aumentar la globalización, las tasas de emigración disminuyen y las de inmigración aumentan (SELA, 2010) estos resultados confirman lo encontrado en este estudio.
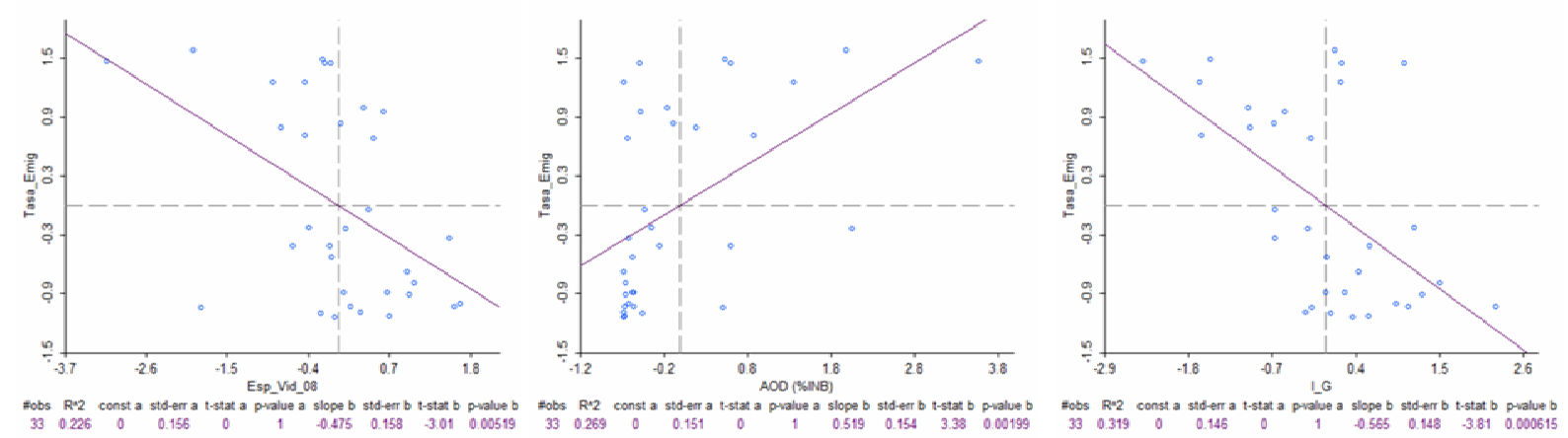

Figura 4. Gráfico de dispersión que muestra el grado de correlación entre los indicadores seleccionados (Esperanza de vida; Ayuda oficial al desarrollo e Índice de globalización) y la tasa de emigración calificada para ALC.

Fuente: elaboración propia con el software GeoDA 1.6.6

El objetivo de la segunda sección de los resultados, es destacar en el gráfico y mapa cuales son los países de América Latina y el Caribe que se encuentran en situaciones "más favorable" y "menos favorable" con respecto a las correlaciones ya mencionadas.

En la figura 5, se representan los resultados de la primera correlación realizada (esperanza de vida al nacer con la tasa de emigración calificada), se observa claramente que la mejor situación (cuadrante IV: espacio +-) corresponde a los países (13 unidades espaciales de 33) localizados al oeste del sub-continente Suramericano (Argentina, Chile, Colombia, Ecuador, Perú, Uruguay y Venezuela) sin contigüidad espacial países de Centroamérica (Costa Rica, México, Nicaragua y Panamá) y el norte del Caribe (Bahamas y Cuba) en estos países los valores de esperanza de vida al nacer son altos y la tasa de emigración calificada baja. En cuanto a la peor situación (cuadrante II: espacio -+), corresponde a dos países al norte de Suramérica (Guyana y Surinam) y un grupo de países caribeños(Dominica, Granada, Haití, San Cristóbal y Nieves, Trinidad y Tobago, San Vicente y Granadinas y Jamaica);(9 unidades espaciales de 33), en este caso cuando los valores de esperanza de vida al nacer son bajos la tasa de emigración calificada es alta.

Publicado en formato digital: Mg. Lcdo. Eloy Montes Galbán. EMIGRACIÓN DE RECURSOS HUMANOS CALIFICADOS Y DESARROLLO EN AMÉRICA LATINA Y EL CARIBE: UNA MIRADA DESDE LA GEOGRAFÍA. Revista Geográfica Digital. IGUNNE. Facultad de Humanidades. UNNE. Año 11. № 22. Julio Diciembre. 2014. ISSN 1668-5180 Resistencia, Chaco.

En: http://hum.unne.edu.ar/revistas/geoweb/default.htm 

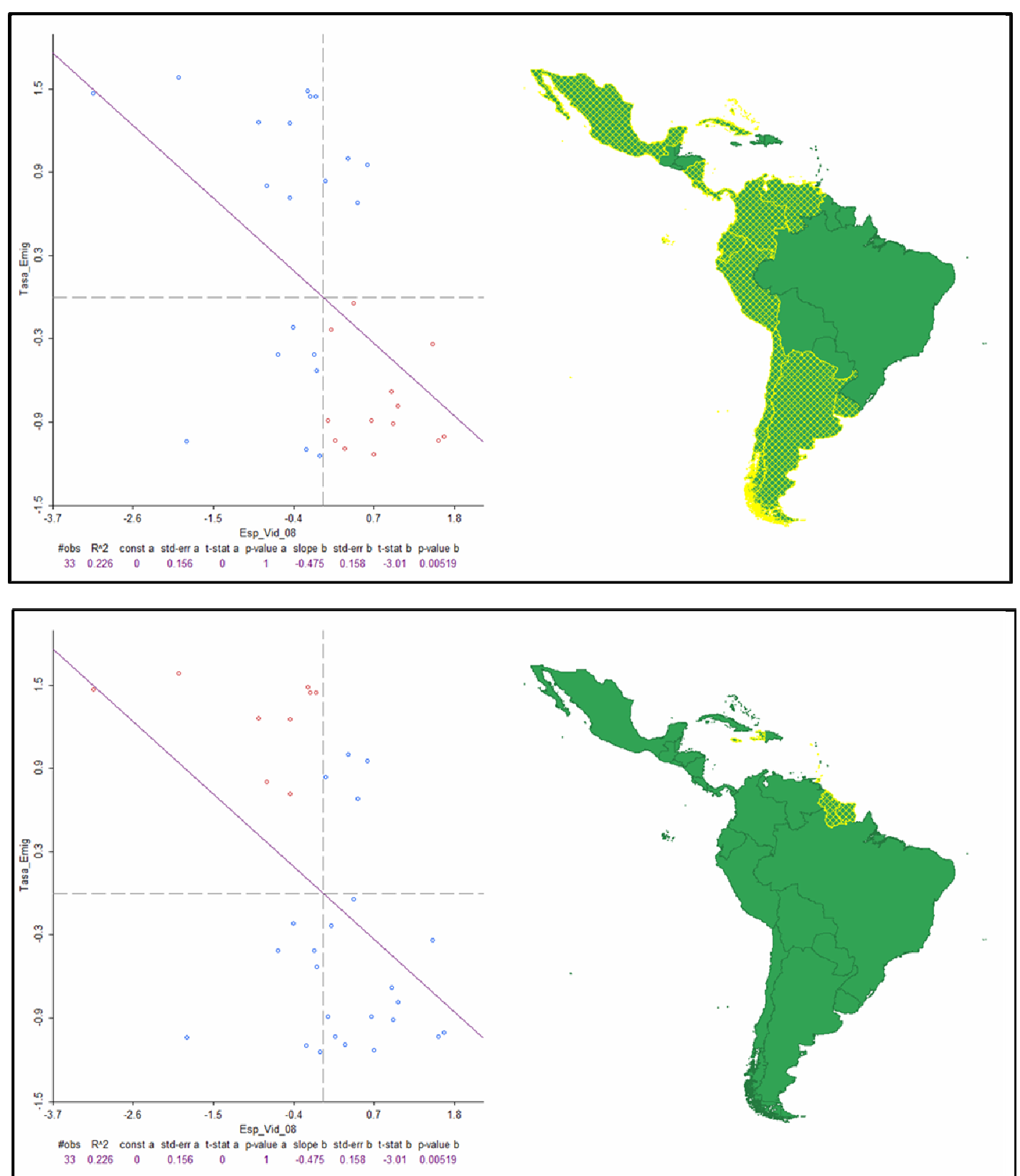

Figura 5. Gráfico y mapa que muestra la relación bivariada entre la esperanza de vida al nacer y la tasa de emigración calificada.

Fuente: elaboración propia con el software GeoDA 1.6.6

En la figura 6, se representan los resultados de la segunda correlación realizada (Ayuda Oficial al Desarrollo neta recibida con la tasa de emigración calificada), se observa claramente que la mejor situación (cuadrante I: espacio - -) corresponde a una gran mayoría de los países estudiados (17 unidades espaciales de 33) presentando la siguiente configuración espacial: la mayoría de los países del sub-continente Suramericano (Argentina, Brasil, Chile, Colombia, Ecuador, Paraguay, Perú, Uruguay y Venezuela), de Centroamérica Costa Rica, El Salvador, Guatemala, México, Panamá y del Caribe: Bahamas, Cuba, República Dominicana, en este grupo de países los valores de la Ayuda

Publicado en formato digital: Mg. Lcdo. Eloy Montes Galbán. EMIGRACIÓN DE RECURSOS HUMANOS CALIFICADOS Y DESARROLLO EN AMÉRICA LATINA Y EL CARIBE: UNA MIRADA DESDE LA GEOGRAFÍA. Revista Geográfica Digital. IGUNNE. Facultad de Humanidades. UNNE. Año 11. № 22. Julio Diciembre. 2014. ISSN 1668-5180 Resistencia, Chaco.

En: http://hum.unne.edu.ar/revistas/geoweb/default.htm 
Oficial al Desarrollo neta recibida son bajos, la tasa de emigración calificada también es baja. En cuanto a la peor situación (cuadrante III: espacio ++), corresponde a dos países al norte de Suramérica (Guyana y Surinam) y un grupo de países caribeños (Dominica, Granada, Haití, San Cristóbal y Nieves, San Vicente y Granadinas) en este caso cuando los valores de la Ayuda Oficial al Desarrollo neta recibida son altos la tasa de emigración calificada también es alta.
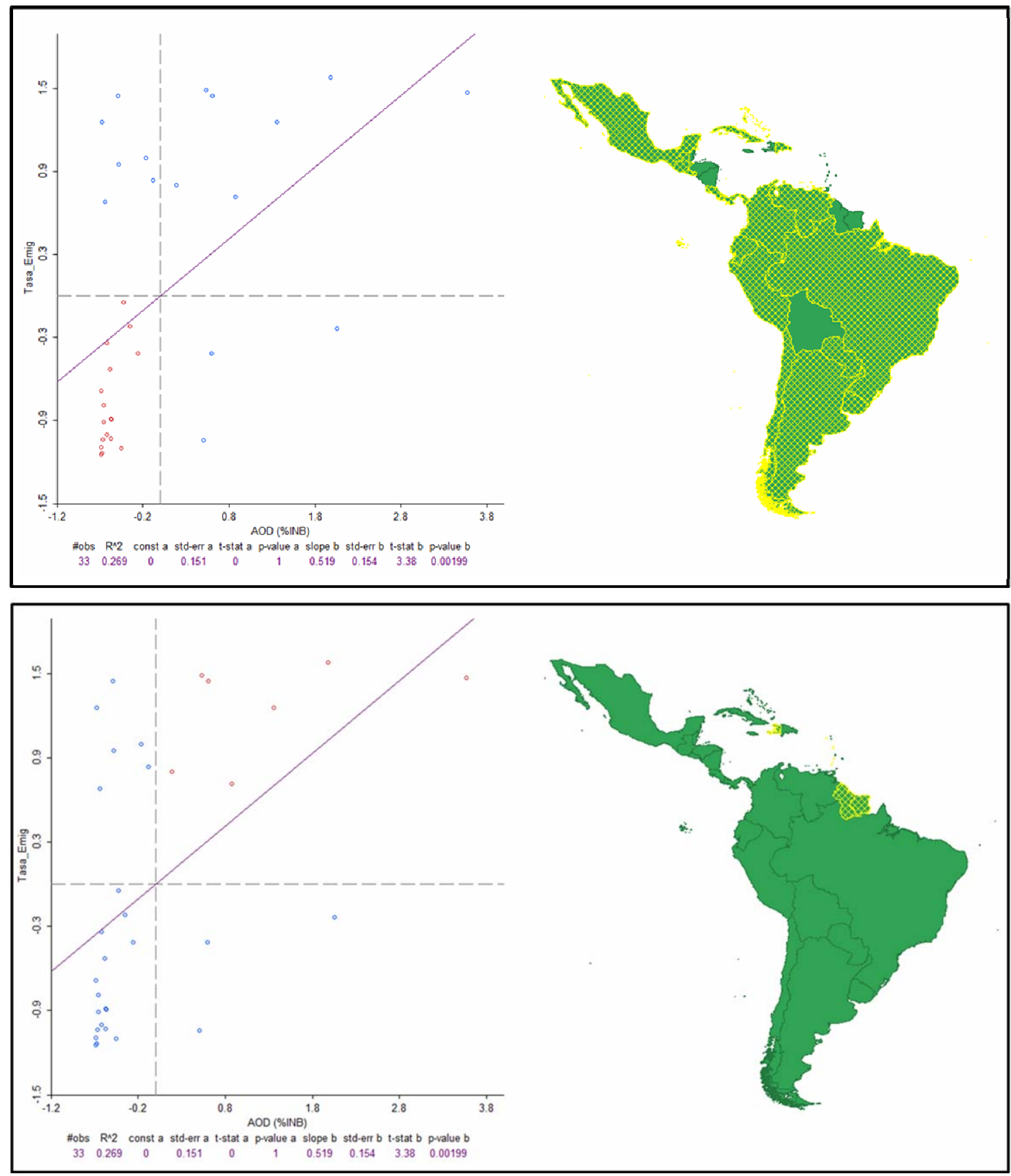

Figura 6. Gráfico y mapa que muestra la relación bivariada entre la Ayuda Oficial al Desarrollo (ODA) neta recibida y la tasa de emigración calificada.

Fuente: elaboración propia con el softwareGeoDA 1.6.6

Publicado en formato digital: Mg. Lcdo. Eloy Montes Galbán. EMIGRACIÓN DE RECURSOS HUMANOS CALIFICADOS Y DESARROLLO EN AMÉRICA LATINA Y EL CARIBE: UNA MIRADA DESDE LA GEOGRAFíA. Revista Geográfica Digital. IGUNNE. Facultad de Humanidades. UNNE. Año 11. № 22. Julio Diciembre. 2014. ISSN 1668-5180 Resistencia, Chaco.

En: http://hum.unne.edu.ar/revistas/geoweb/default.htm 
En la figura 7, se muestran los resultados de la tercera correlación realizada (Índice de globalización con la tasa de emigración calificada), se logra apreciar que la mejor situación (cuadrante IV: espacio +-) corresponde a los países (15 unidades espaciales de 33) localizados en casi la totalidad del sub-continente Suramericano (Argentina, Brasil, Chile, Colombia, Ecuador, Paraguay,
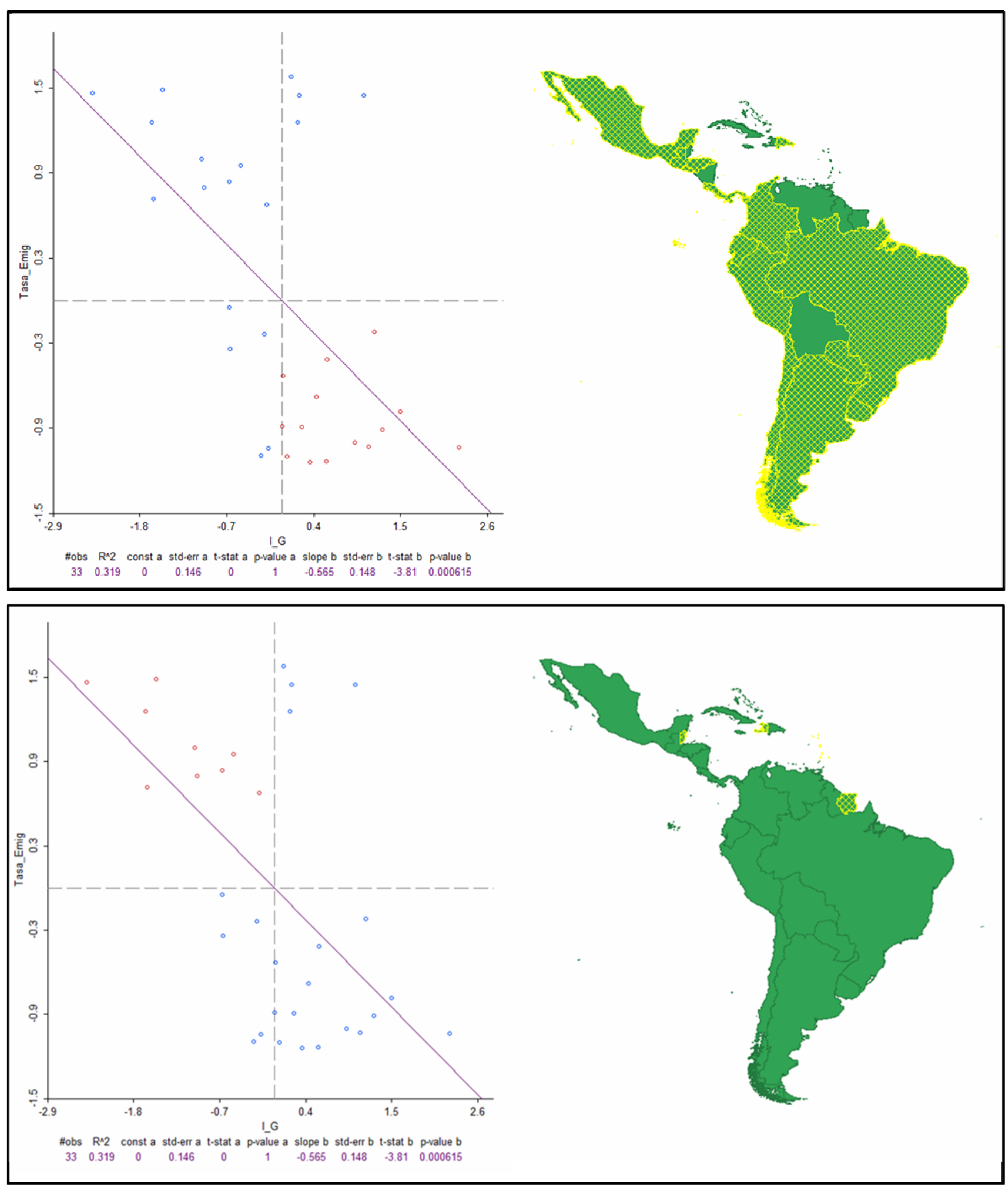

Figura 7. Gráfico y mapa que muestra la relación bivariada entre el índice de globalización y la tasa de emigración calificada.

Fuente: elaboración propia con el software GeoDA 1.6.6

Publicado en formato digital: Mg. Lcdo. Eloy Montes Galbán. EMIGRACIÓN DE RECURSOS HUMANOS CALIFICADOS Y DESARROLLO EN AMÉRICA LATINA Y EL CARIBE: UNA MIRADA DESDE LA GEOGRAFÍA. Revista Geográfica Digital. IGUNNE. Facultad de Humanidades. UNNE. Año 11. № 22. Julio Diciembre. 2014. ISSN 1668-5180 Resistencia, Chaco.

En: http://hum.unne.edu.ar/revistas/geoweb/default.htm 
Perú, Uruguay),en Centroamérica: Costa Rica, Guatemala, Honduras, México, Panamá y El Salvador, en el Caribe solo República Dominicana, en estos países los valores del índice de globalización son altos y la tasa de emigración calificada baja. En cuanto a la situación menos favorable (cuadrante II: espacio -+), corresponde a (9 unidades espaciales de 33) un país ubicado al norte del sub-continente Suramérica (Surinam), en Centroamérica Belice y un grupo de países caribeños (Antigua y Barbudas, Barbados, Dominica, Haití, San Cristóbal y Nieves, Santa Lucia, San Vicente y Granadinas) en este caso cuando los valores de índice de globalización son bajos la tasa de emigración calificada es alta.

\section{Consideraciones finales}

Entre las principales conclusiones que se pueden derivar del presente trabajo están: en cuanto a la relación entre el índice de esperanza de vida al nacer y la tasa de emigración calificada en ALC, está clara la tendencia que a menor índice de esperanza de vida al nacer mayor tasa de emigración calificada. En cuanto a la relación entre la Ayuda Oficial al Desarrollo neta recibida con la tasa de emigración calificada la correlación es positiva, confirmando los hallazgos que la emigración calificada no es solo el resultado de la falta de desarrollo económico de los países de origen, sino que también el desarrollo económico en muchos casos puede convertirse en un estímulo para los procesos migratorios. De la tercera correlación realizada, se concluye que a menores niveles en los índices de globalización mayores tasas de emigración calificada.

Los países de la región ALC que tienen una situación más favorable con respecto a los tres indicadores correlacionados con la tasa de emigración calificada son: Argentina, Chile, Colombia, Ecuador, Perú, Uruguay, Costa Rica, México y Panamá. Asimismo, los que presentan una situación menos favorable son: Surinam, Dominica, Haití, San Cristóbal y Nieves, San Vicente y Granadinas.

Este trabajo constituye una aproximación al tema de la emigración calificada en ALC, sin embargo continúa siendo fundamental el contar con estadísticas actualizadas sobre el tema, cosa a lo que muchos de los gobiernos de países de la región no contribuyen, pues no publican con un nivel de confiabilidad aceptable los datos necesarios para el desarrollo de este tipo de estudios. Sin querer redundar en la importancia que tienen este tipo de trabajos, se debe destacar que el análisis espacial a través del Análisis Exploratorio de Datos Espaciales (ESDA) y el posterior análisis geográfico pueden brindar luces que permitan soportar la toma de decisiones al momento de generar políticas migratorias y de desarrollo humano.

(1) Una de las principales características que presenta las migraciones internacionales en la actualidad puede ser resumida en el término "mundialización de las migraciones", el mapa de los flujos migratorios ha cambiado, con la diversificación de rutas y conexiones de origen -destino, hasta hace solo medio siglo los países receptores por excelencia eran Estados Unidos, Canadá, Argentina, Brasil y Australia y el grueso de las población emigrante tenía como origen Europa; en la actualidad los flujos de origen pueden provenir de Asia, África y América Latina convirtiéndose en nuevos destinos algunos países del Golfo Pérsico y Europa.

(2) La Organización para la Cooperación y el Desarrollo Económicos (OCDE) es un organismo de cooperación internacional, fundado en 1961, agrupa a 34 países miembros y su misión es promover políticas que mejoren el bienestar económico y social de las personas alrededor del mundo.

(3) Software desarrollado por el Beoda Center for Geospatial Analysis and Computation de la Universidad del estado de Arizona, actualmente la versión disponible del programa es la 1.6.6

Publicado en formato digital: Mg. Lcdo. Eloy Montes Galbán. EMIGRACIÓN DE RECURSOS HUMANOS CALIFICADOS Y DESARROLLO EN AMÉRICA LATINA Y EL CARIBE: UNA MIRADA DESDE LA GEOGRAFíA. Revista Geográfica Digital. IGUNNE. Facultad de Humanidades. UNNE. Año 11. № 22. Julio Diciembre. 2014. ISSN 1668-5180 Resistencia, Chaco. 
Revista Geográfica Digital. IGUNNE. Facultad de Humanidades. UNNE. Año 11. № 22. Julio - Diciembre 2014. ISSN 1668-5180 Resistencia, Chaco

\title{
Referencias citadas
}

Banco Mundial (2008) Indicador: Ayuda Oficial al Desarrollo (ODA) neta recibida (\% del INB). Recuperado de: http://datos.bancomundial.org/indicador

Banco Mundial (2008) Indicador: esperanza de vida al nacer, total (años).Recuperado de: http://datos.bancomundial.org/indicador

Belloc, Filippo (2008) International Economic Assistance and Migration: The Case of SubSaharan Countries. MPRA Paper No. 17290, posted 14. Recuperado en: http://mpra.ub.uni$\underline{\text { muenchen.de/17290/ }}$

\author{
Banco Mundial (2009)World Development report 2009: Reshaping Economic Geography. \\ Washington $D C$ : Banco Mundial. \\ en:http://siteresources.worldbank.org/INTWDR2009/Resources/4231006- \\ 1225840759068/WDR09 bookweb 2.pdf
}

Bosque Sendra, Joaquín y Moreno Jiménez, Antonio. (1994) Prácticas de análisis exploratorio y multivariante de datos. Oikos-tau. Barcelona, España.

Buzai, Gustavo y Baxendale, Claudia (2009) Análisis exploratorio de datos espaciales. Geografía y Sistemas de Información Geográfica. (GESIG-UNLU, Luján). Año 1, № 1, Sección III:1-11. Disponible en: www.gesig-proeg.com.ar

Buzai, Gustavo y Baxendale, Claudia (2012) Análisis socioespacial con Sistemas de Información Geográfica. Ordenamiento territorial, temáticas de base vectorial (Primera ed., Vol. II). Lugar Editorial, Buenos Aires, Argentina.

Buzai, Gustavo; Baxendale, Claudia; Principi, Noelia; Cruz, María del Rosario; Cacace, Graciela; Caloni, Nicolás; Humataca, Luis; Mora, Jimena y Paso Viola, Fernando (2013) Sistemas de Información Geográfica (SIG): teoría y aplicación. Universidad Nacional de Luján, Luján, Argentina.

Cerezo, Yakary; Romero, Adelmo y Montes, Eloy (2013) Uso de las tecnologías de la información geográfica para la optimización de la eficiencia y justicia espacial. Hélice: Revista Venezolana de Ciencias de la Información. Vol.5 ( $\left.N^{\circ} 1\right)$, enero-junio. Universidad del Zulia.

Comisión Económica para América Latina y el Caribe (2014) La Inversión Extranjera Directa en América Latina y el Caribe. Informe 2013. Santiago de Chile, publicación de las Naciones Unidas. Recuperado de: http://www.cepal.org/publicaciones/xml/9/52979/lainversionextranjera2013.pdf

Docquier, F., B. Lindsay Lowell y A. Marfouk (2009) A Gendered Assessment of Highly Skilled Emigration. Population and DevelopmentReview Vol. 35, Núm. 2 Junio2009. Recuperado en: http://perso.uclouvain.be/frederic.docquier/filePDF/DataSetByGender Aggregates.xls

GeoDa Center for Geospatial Analysis and Computation Development Team. (2014) GeoDa Introduction to Spatial Data Analysis: EDA, ESDA \& ML Spatial Regression, versión 1.6.6. Disponible en: $\underline{\text { http://geodacenter.asu.edu/software/downloads }}$

Hernández S., Roberto; Fernández, Carlos y Baptista, Pilar (2003) Metodología de la investigación. Tercera Edición McGraw-Hill. México.

Publicado en formato digital: Mg. Lcdo. Eloy Montes Galbán. EMIGRACIÓN DE RECURSOS HUMANOS CALIFICADOS Y DESARROLLO EN AMÉRICA LATINA Y EL CARIBE: UNA MIRADA DESDE LA GEOGRAFíA. Revista Geográfica Digital. IGUNNE. Facultad de Humanidades. UNNE. Año 11. № 22. Julio Diciembre. 2014. ISSN 1668-5180 Resistencia, Chaco.

En: http://hum.unne.edu.ar/revistas/geoweb/default.htm 
Instituto de investigación económica Konjunkturforschungsstelle KOF (2011) KOF Index of Globalization 2011.Recuperado de: http://globalization.kof.ethz.ch/

Martínez Pizarro, Jorge (2006)Globalizados, pero restringidos. Una visión del mercado global de recursos humanos calificados, en Alejandro I. Canales (editor), Panorama actual de las migraciones en América Latina, México, Universidad deGuadalajara.

Natural Earth. Cultural vector countries [Capa vectorial]. 1:10 m. "There are 247 countries in theworld". Versión 3.1.0. Disponible en: http://www.naturalearthdata.com/downloads/10m-cultural-vectors/. (1 de octubre de 2014).

Programa de Naciones Unidas para el Desarrollo (2014) Human Development Index trends, 19802013. Recuperado de: http://hdr.undp.org/es/content/table-2-human-development-index-trends-1980$\underline{2013}$

Quantum GIS DevelopmentTeam. (2014). Quantum GIS GeographicInformationSystem. Open Source Geospatial Foundation Project. Recuperado de http://qgis.osgeo.org.

Sistema de Económico Latinoamericano y del Caribe (2009) La emigración de recursos humanos calificados desde países de América Latina y el Caribe Tendencias contemporáneas y perspectivas. $\quad$ Recuperado de: http://www.sela.org/attach/258/EDOCS/SRed/2009/09/T023600003722-0La emigracion de recursos humanos calificados desde ALC.pdf

Sistema de Económico Latinoamericano y del Caribe (2010) Migración de Recursos Humanos Calificados $y$ Desarrollo Humano en América Latina $y$ el Caribe. Recuperado de:http://www.sela.org/attach/258/EDOCS/SRed/2010/10/T023600004391-0Migracion de Recursos Humanos Calificados en ALC.pdf

Publicado en formato digital: Mg. Lcdo. Eloy Montes Galbán. EMIGRACIÓN DE RECURSOS HUMANOS CALIFICADOS Y DESARROLLO EN AMÉRICA LATINA Y EL CARIBE: UNA MIRADA DESDE LA GEOGRAFÍA. Revista Geográfica Digital. IGUNNE. Facultad de Humanidades. UNNE. Año 11. No 22. Julio Diciembre. 2014. ISSN 1668-5180 Resistencia, Chaco.

En: http://hum.unne.edu.ar/revistas/geoweb/default.htm 